\title{
Development of a Modified Disability Questionnaire for Evaluating Disability Caused by Backache in India and Other Developing Countries
}

\author{
Janardhana P. Aithala ${ }^{1}$, Suraj Kumar ${ }^{2}$, Shodhan Aithal ${ }^{2}$, Shashidhar M. Kotian ${ }^{2}$ \\ ${ }^{1}$ Department of Orthopedics, Yenepoya Medical College, Mangalore, India \\ ${ }^{2}$ Kasturba Medical College, Mangalore, India
}

\section{Study Design: Prospective observational study.}

Purpose: To evaluate the disability domains relevant to Indian patients with low backache and propose a modified disability questionnaire for such patients.

Overview of Literature: The Oswestry Disability Index (ODI) is a self-reported measurement tool that measures both pain and functional status and is used for evaluating disability caused by lower backache. Although ODI remains a good tool for disability assessment, from the Indian perspective questions related to weight lifting and sexual activity of ODI are questioned in some of the earlier studies. Activities of daily living in Indian patients vary substantially from those in other populations and include activities like bending forwards, sitting in floor and squatting which are not represented in the ODI.

Methods: In this prospective observational study, a seven-step approach was used for the development of a questionnaire. Thirty patients were interviewed to identify the most challenging issue they faced while performing their daily activities (by free listing) and understand how important the questionnaire items were in terms of the standard ODI. Thus, a comprehensive disability questionnaire comprising 14 questions was developed and administered to 88 patients. Both qualitative (interviews) and quantitative methods (to establish the validity, reliability, and correlation with the Visual Analog Scale [VAS] and Rolland Morris disability questionnaire) were used to identify the 10 questions that best addressed the disability domains relevant to Indian patients.

Results: According to free listing, four new questions pertaining to bending forward, sitting on the floor, walking on uneven surfaces, and work-related disabilities were included. In the second phase, wherein the questionnaire with 14 items was used, 56.8\% patients did not answer the questions related to sexual activity, whereas $23.8 \%$ did not answer those related to walking on uneven surfaces. The modified questionnaire demonstrated good internal consistency (Cronbach's alpha=0.892) and correlation with the Rolland Morris questionnaire (Cronbach's alpha $=0.850, p>0.05$ ), as well as with the VAS score for disability (Cronbach's alpha=0.712, $p>0.05$ ) and pain (Cronbach's alpha=0.625, $p>0.05$ ).

Conclusions: A modified disability questionnaire that was designed by adding two questions related to bending forward and work status and removing questions related to sexual activity and weight lifting or traveling (depending on the occupation) can help evaluate disability caused by back pain in Indian population.

Keywords: Spine; Back pain; Oswestry Disability Index; Surveys and questionnaire

Received Jan 6, 2018; Revised Apr 9, 2018; Accepted Apr 29, 2018

Corresponding author: Suraj Kumar

Kasturba Medical College, Mangalore, India

Tel: +91-890-4529437, Fax: +91-824-2428183, E-mail: samkumar890@gmail.com 


\section{Introduction}

The Oswestry Disability Index (ODI) is a self-reported measurement tool that measures both pain and functional status and is used for evaluating disability caused by lower backache $[1,2]$. This index was developed in 1980 by Fairbank et al. [1] and is one of the most commonly used disability scales [3-5]. This scoring tool has been tested for its reliability and efficacy [6,7]. The ODI comprises 10 domains that evaluate the disability of a patient; these domains are based on pain intensity, personal care, lifting, walking, sitting, standing, sleeping, sex life, social life, and traveling. While the ODI remains an excellent tool for evaluating disability caused by backache and treatment outcome, a few items may not actually contribute or help in disability assessment. Several studies have demonstrated that the sexual activity domain of the ODI remained unanswered by a majority of the population [8]. Various modified versions of the ODI have been developed [9-12]; Hudson-Cook et al. [9] published a modified ODI wherein the sexual activity domain was replaced by changing degrees of pain. Fritz and Irrgang [10] developed a modified ODI that replaced the sexual activity domain with employment/homemaking. Similarly, the American Academy of Orthopaedic Surgeons devised a spine outcome instrument by deleting domains 1,8 , and 9 of the standard ODI [12]. From the Indian perspective, Nishant et al. [13] demonstrated that the sexual activity domain of the ODI remains unanswered by a majority of the population. Questions regarding weight lifting were also criticized because most patients may not perform this activity in their daily routine [14]. Further, there is a possibility that the actual performance differs from the patient's answer [7].

As reported in various studies, activities of daily living in Indian patients vary substantially from those in other populations. Indian patients need to sit cross-legged on the floor, bend forward, squat, and walk on uneven surfaces $[15,16]$. Scales, such as the Quebec back pain disability scale and the Rolland Morris disability questionnaire (RMDQ) address the issues related to bending forward and stair climbing $[17,18]$; however, these activities are not represented in the ODI; thus, the use of the ODI in Indian patients may not address all the domains of their disability. These limitations were highlighted in a study on the validation of translator-assisted scoring that has identified some difficulties in scoring the ODI and stated that if these issues are addressed [14], the ODI can be a very good tool for disability assessment in Indian patients. Hence, this study was planned to identify the domains that are relevant to Indian patients and then modify the ODI to enable its use in Indian patients.

The modification of the ODI and the identification of the components or domains required for inclusion in the ODI are challenging. Our study aimed to study and understand the various disability domains relevant to Indian patients and to determine which ODI questions are relevant in the Indian setup. This would serve as a first step toward the formation of a questionnaire that requires pilot testing. We believe that the results of the present study will enable us to design a 'backache disability questionnaire' suitable for patients in India and other developing countries with similar socioeconomic and cultural background.

\section{Materials and Methods}

This prospective observational study was performed at the Kasturba Medical College, Mangalore, India from 2015 to 2016; ethical committee clearance was obtained from the Institutional Ethics Committee (IEC KMC MLR 05-15/115). Informed consent was taken from all the participants. The questionnaire was administered to patients who presented with low back pain to the outpatient department (orthopedics) of a tertiary care center and were able to understand and answer the questionnaire items. Patients with infections, tumors, and pathological fractures as well as those unable to walk due to gross neurological deficits were excluded from the study.

A seven-step approach to questionnaire development by Gehlbach et al. [19] in 2010 was used, with some modifications. These steps include the following: (1) literature review/need analysis, (2) interviews and focus group discussions, (3) synthesis of the information obtained from second step, (4) questionnaire development, (5) expert validation, (6) personal interviews to ensure that the respondents interpret the items in questionnaire correctly, (7) and pilot test. The first step, including the need for modification, was addressed in our introduction with a brief review of the literature. The second step of our study included interviews of 30 patients (purposive sampling) who presented to the outpatient department with low back pain. We used free listing for the most important activity/activities that were compromised by the back pain experienced by these patients. Each participant was asked 
to list all the activities affected by back pain that lead to disability. In addition, each patient was asked to report whether the activities mentioned in the standard ODI were relevant to their routine activities. With this step, we tried to identify the disability domains in patients with back pain. Free listing, response to qualitative questions asked for each standard ODI item, and the information available in the literature were used to develop additional items (synthesis of information: 3rd step).

A new modified questionnaire was developed (4th step) by adding four new questions. Expert validation (5th step) was performed by the senior author and the statistician. The 6th and 7th steps were clubbed together, and the new extended ODI with four additional items was administered to patients presenting with low back pain in the outpatient department along with the standard ODI, Visual Analog Scale (VAS) for pain, VAS for disability, and RMDQ [20]. The study population comprised Kannadaspeaking individuals; therefore, a Kannada language questionnaire of the original and the modified ODI was developed by forward translation into the Kannada language by expert translators followed by back translation into English by two other translators; a pre-final version was decided after a review by the authors. The Kannada version was separately tested for internal consistency and reliability using Cronbach's alpha. Detailed statistical analyses were performed using appropriate statistical tests to determine the patients' response to the items, correlation between the modified and standard ODIs as well as the RMDQ, and internal validity using Cronbach's alpha. Using these statistical tests, the questionnaire was reevaluated, and a 10-question modified backache disability questionnaire was proposed that needed to be tested for validity, reliability, and usefulness in predicting the treatment response through multi-centric studies for adaptation in daily practice.

\section{Results}

\section{Developing questionnaire items}

In order to modify the questionnaire for Indian subjects, we collected qualitative data from 30 participants regarding daily activities affected by back pain, using free listing. We used purposive sampling and the occupation profile of these patients, including 12 laborers, six marketing executives, five office workers, six household workers, and one
Table 1. Frequency of day to day activities affected by backache from free listing $(\mathrm{N}=30)$

\begin{tabular}{|llr|}
\hline Serial no. & \multicolumn{1}{c}{ Activities } & $\begin{array}{c}\text { Frequency } \\
(\%)\end{array}$ \\
\hline 1 & Bending forwards & $20(67)$ \\
\hline 2 & Sitting cross legged and squatting & $16(53)$ \\
\hline 3 & Walking & $11(37)$ \\
\hline 4 & Getting up from bed, from sitting position & $9(30)$ \\
\hline 5 & Weight lifting & $8(27)$ \\
\hline 6 & House hold work & $5(17)$ \\
\hline 7 & Sitting & $5(17)$ \\
\hline 8 & Standing & $5(17)$ \\
\hline 9 & Walking in uneven surface or climbing & $4(13)$ \\
\hline 10 & Stairs & $3(10)$ \\
\hline
\end{tabular}

person leading a retired life. Table 1 shows the details of free listing.

Interviewed participants $(n=30)$ were asked about the requirement of each ODI item in their daily life. Majority $(60 \%, \mathrm{n}=18)$ felt that sexual activity (ODI-8) was not necessary in their daily life. Lifting weights $(16 \%, n=5)$, traveling $(20 \%, n=6)$, social life $(10 \%, n=3)$, personal care $(3 \%, \mathrm{n}=1)$, walking $(3 \%, \mathrm{n}=1)$, standing $(3 \%, \mathrm{n}=1)$, and sleeping $(3 \%, \mathrm{n}=1)$ were the other activities that were reportedly unimportant in the routine life of the study participants.

Based on the details obtained from free listing and a previous study by the senior author [14], four activities were added to the standard ODI. The modified ODI included bending forward (ODI 11), sitting on the floor (ODI 12), walking on an uneven surface (ODI 13), and work status (ODI 14) [21]. The response items were carefully written after discussion with other subject experts who work in spine care, based on reference articles, and in consultation with some patients, depending on the disability severity. In the initial few patients, a trial assessment was performed using these new questions to ensure that the grading of the questionnaire items was in a manner that showed progressively increasing disability levels and correlated with the responses to the standard ODI questionnaire. This helped to modify the questionnaire responses slightly before the pilot testing. Correlation analysis with the standard ODI questionnaire scores was also performed at study completion. Along with new questions, two qualitative questions were asked for each 
of ODI question: one, whether the response to the activity was influenced by any other reason except back pain; second, whether the mentioned activity is required in their daily activities. The following four questions added:

\section{1) Bending forward}

Questions are as follows: comfortable while picking up objects from the floor by bending the back and comfortably performing tasks that require bending forward; can bend and pick up objects from the floor, but cannot do it consistently because of pain; can bend and pick up objects placed at knee level, bending beyond that caused pain; can bend and pick up objects placed at waist level comfortably, bending more than that causes pain; can bend and pick up objects placed at waist level with difficulty; and cannot bend at all.

\section{2) Sitting on the floor}

Questions are as follows: can sit on the floor for as long as I like and can squat comfortably; can sit on the floor for up to 2 hours; thereafter, I need back support; can sit for up to 1 hour; can sit for up to half an hour only; cannot sit for more than 10 minutes; and cannot sit on the floor at all.

\section{3) Walking on an uneven surface}

Questions are as follows. Pain does not prevent me from walking for more than 1 hour on uneven surfaces, such as fields. Pain prevents me from walking for more than 30 minutes on uneven surfaces. Pain prevents me from walking for more than 10 minutes. Pain prevents me from walking on uneven surfaces; however, I can walk for more than 10 minutes on level surfaces. Pain prevents me from walking on level surfaces also for more than 10 minutes. Pain prevents me from walking at all.

\section{4) Work status}

Questions are as follows: able to do gainful work/household work; able to do work, but with some pain and medicines; able to do work, but with pain and medicines; need to take leaves occasionally; irregular at work because of pain; on loss of pay, but able to do limited household work, including personal care; and unable 'to do personal work and am dependent for it.

\section{Assessing the validity and reliability of the new ques- tionnaire and developing a final questionnaire}

Total 88 patients (sample size calculated with a reliability threshold of 0.754) were now included in the next part of the study to pilot test the new questionnaire. Both standard and extended ODI scores were calculated; the extended ODI was calculated by including total 14 questions and then adjusting the score to 100 (actual score $\times 20$ / number of items answered by the patient).

Mean standard ODI and extended ODI were $41.19 \pm 20.04$ and $43.92 \pm 20.58$, respectively. The mean age of the study population was $50.5 \pm 14.5$ years. The mean body mass index was $23.98 \pm 4.1 \mathrm{~kg} / \mathrm{m}^{2}$. Forty-five participants were women (51\%). Majority of the patients had degenerative disk disease $(56.8 \%, \mathrm{n}=50)$ followed by mechanical backache $(25 \%, \mathrm{n}=22)$, lumbar spondylolisthesis $(9.1 \%, \mathrm{n}=8)$, lumbar canal stenosis $(6.8 \%, \mathrm{n}=6)$, and old healed fracture $(2.3 \%, n=2)$. Nearly $36.4 \%(n=32)$ were unable to perform their routine duties owing to low back pain. Most of the subjects were manual laborers ( $37.5 \%, n=33)$, followed by house makers $(28.4 \%, n=25)$, and office workers $(12.5 \%, \mathrm{n}=11)$. The median duration of low back pain among the participants was 6 months (interquartile range, 1.25-36 months). The collected data were self-reported in $55.7 \%(n=49)$ of the participants and translator-assisted in $46.3 \%(\mathrm{n}=39)$ participants.

Among the 88 participants, $54.5 \%(n=48)$ did not answer ODI 8 (sexual activity) and $37.5 \%(\mathrm{n}=33)$ did not answer ODI 13 (walking on uneven surface). We added two extra qualitative questions to the existing 6 points in the ODI; this included questions regarding whether the response to the activity is influenced by any other reason other than backache and whether the activity is relevant to their daily activities. The frequencies of ODI questions left unanswered by the participants and the responses to the two extra questions are shown in Table 2. Lifting weights, sexual activity, traveling, sitting on the floor, and walking on uneven surface were the activities that were considered by several participants as irrelevant to their daily routine. The newly added questions were compared with occupation; Table 3 gives an insight regarding these items. Question number 8 and 13 were not answered by several participants; therefore, we decided to exclude them from the reliability and correlation tests to improve the reliability of these tests. 
Table 2. Frequencies of ODI questions left unanswered and response to additional clauses ( $\mathrm{n}=88$ )

\begin{tabular}{|c|c|c|c|}
\hline ODI & Affected, but not due to backache & Not needed in routine activities & Not answered (\%) \\
\hline ODI 1 (pain intensity) & 1 & - & 0 \\
\hline ODI 2 (personal care) & 0 & 2 & 1 \\
\hline ODI 3 (lifting weights) & 1 & 14 & 5 \\
\hline ODI 4 (walking) & 4 & 2 & 1 \\
\hline ODI 5 (sitting) & 1 & 0 & 0 \\
\hline ODI 6 (standing) & 1 & 2 & 1 \\
\hline ODI 7 (sleep) & 7 & 0 & 1 \\
\hline ODI 8 (sexual activity) & 0 & 48 & $50(56.8)$ \\
\hline ODI 9 (social life) & 2 & 10 & 5 \\
\hline ODI 10 (travel) & 0 & 20 & 8 \\
\hline ODI 11 (bending forwards) & 0 & 5 & 1 \\
\hline ODI 12 (sitting on floor) & 1 & 17 & 11 \\
\hline ODI 13 (walking on uneven surface) & 0 & 33 & $21(23.8)$ \\
\hline ODI 14 (work status) & 0 & 7 & 2 \\
\hline
\end{tabular}

ODI, Oswestry Disability Index.

Table 3. Comparison of occupation with requirement of the newly added activities ( $N=88$ )

\begin{tabular}{|c|c|c|c|c|c|c|c|c|c|c|c|c|}
\hline \multirow[t]{2}{*}{ New ODI questions } & \multicolumn{2}{|c|}{ Clerical work } & \multicolumn{2}{|c|}{ Laborer } & \multicolumn{2}{|c|}{$\begin{array}{c}\text { Marketing } \\
\text { representative }\end{array}$} & \multicolumn{2}{|c|}{ Office work } & \multicolumn{2}{|c|}{ Retired } & \multicolumn{2}{|c|}{ Housewife } \\
\hline & Yes & No & Yes & No & Yes & No & Yes & No & Yes & No & Yes & No \\
\hline \multicolumn{13}{|l|}{ Required in their day to day activities } \\
\hline ODI 11 (bending forwards) & 6 & 1 & 31 & 2 & 4 & 1 & 11 & 0 & 7 & 0 & 24 & 1 \\
\hline ODI 12 (sitting on floor) & 5 & 2 & 28 & 5 & 3 & 2 & 9 & 2 & 6 & 1 & 20 & 5 \\
\hline ODI 13 (walking on uneven surface) & 3 & 4 & 23 & 10 & 4 & 1 & 4 & 7 & 6 & 1 & 15 & 10 \\
\hline ODI 14 (work status) & 7 & 0 & 33 & 0 & 5 & 0 & 10 & 1 & 3 & 4 & 23 & 2 \\
\hline
\end{tabular}

ODI, Oswestry Disability Index.

Table 4. Cronbach's alpha with item deleted for standard ODI and extended ODI

\section{ODI questions Cronbach's alpha with item deleted (standard ODI) Cronbach's alpha with item deleted (extended ODI}

\begin{tabular}{lll}
1 & 0.860 & 0.887 \\
\hline 2 & 0.862 & 0.884 \\
\hline 3 & 0.871 & 0.887 \\
\hline 4 & 0.856 & 0.881 \\
\hline 5 & 0.857 & 0.881 \\
\hline 6 & 0.853 & 0.881 \\
\hline 7 & 0.870 & 0.889 \\
9 & 0.847 & 0.875 \\
\hline 10 & 0.850 & 0.878 \\
\hline 11 & - & 0.892 \\
\hline 14 & - & 0.888 \\
\hline
\end{tabular}

ODI, Oswestry Disability Index. 


\section{Reliability analyses using Cronbach's alpha}

Cronbach's alpha score of the standard ODI, after excluding ODI 8 (concerning sexual activity) was 0.873 (nine items). The modified ODI had a Cronbach's alpha score of 0.892 (12 items) after ODI 8 (sexual activity) and ODI 13 (walking on uneven surface) were removed. The Kannada language questionnaire was answered by 22 participants, while the remaining patients self-reported in the English version. Cronbach's alpha with the deleted items for the standard and modified ODIs is shown in Table 4. A separate reliability analysis was performed for the Kannada version of the ODI using Cronbach's alpha and was found to be 0.918 and 0.960 for the standard and modified versions of the ODI, respectively, indicating good internal consistency.

\section{Correlation of the ODI items with standard and ex- tended ODI, RMDQ, and VAS score for pain and dis- ability}

Spearman's correlation and Kendal tau correlation between individual the ODI items with standard ODI, extended ODI, RMDQ, VAS score for pain and disability were good for all the items and are shown in Table 5. Questions related to sexual function and walking on uneven surface were omitted because these questions were not answered by many and could affect the reliability of the tests. A comparison of the standard ODI and modified ODI in relation to the mean and correlation with the Roland Morris score and VAS for pain and disability is shown in Table 6.

Questions 5 and 12 overlapped slightly; therefore, responses to ODI 5 (sitting) and ODI 12 (sitting on the

Table 5. Spearman's and Kendal Tau correlation coefficient comparing ODI items with standard ODI, modified ODI score, Rolland Morris disability questionnaire and Visual Analog Scale score

\begin{tabular}{|c|c|c|c|c|c|c|c|c|}
\hline \multirow{2}{*}{$\begin{array}{l}\text { ODI } \\
\text { questions }\end{array}$} & \multicolumn{2}{|c|}{ Standard ODI } & \multicolumn{2}{|c|}{ Modified questionnaire } & \multicolumn{2}{|c|}{$\begin{array}{c}\text { Rolland Morris disability } \\
\text { questionnaire }\end{array}$} & \multicolumn{2}{|c|}{ Visual Analog Scale score } \\
\hline & Spearman & Kendal Tau & Spearman & Kendal Tau & Spearman & Kendal Tau & Spearman & Kendal Tau \\
\hline 1 & 0.697 & 0.566 & 0.637 & 0.502 & 0.586 & 0.451 & 0.722 & 0.636 \\
\hline 2 & 0.604 & 0.481 & 0.610 & 0.483 & 0.572 & 0.455 & 0.344 & 0.286 \\
\hline 3 & 0.629 & 0.477 & 0.630 & 0.487 & 0.550 & 0.430 & 0.388 & 0.302 \\
\hline 4 & 0.715 & 0.594 & 0.707 & 0.583 & 0.693 & 0.564 & 0.377 & 0.293 \\
\hline 5 & 0.709 & 0.559 & 0.718 & 0.563 & 0.565 & 0.440 & 0.377 & 0.294 \\
\hline 6 & 0.780 & 0.637 & 0.744 & 0.600 & 0.562 & 0.446 & 0.427 & 0.351 \\
\hline 7 & 0.533 & 0.419 & 0.456 & 0.354 & 0.359 & 0.275 & 0.354 & 0.281 \\
\hline 9 & 0.796 & 0.652 & 0.788 & 0.641 & 0.695 & 0.558 & 0.460 & 0.367 \\
\hline 10 & 0.813 & 0.681 & 0.796 & 0.657 & 0.691 & 0.566 & 0.594 & 0.492 \\
\hline 11 & 0.432 & 0.335 & 0.566 & 0.452 & 0.503 & 0.398 & 0.386 & 0.308 \\
\hline 12 & 0.544 & 0.425 & 0.674 & 0.539 & 0.522 & 0.409 & $0.227(p=0.047)$ & $0.172(p=0.054)$ \\
\hline 14 & 0.678 & 0.540 & 0.779 & 0.635 & 0.711 & 0.566 & 0.491 & 0.393 \\
\hline
\end{tabular}

ODI, Oswestry Disability Index.

Table 6. Correlation between standard ODI and extended ODI

\begin{tabular}{lcc} 
Variable & Modified ODI & Standard 0DI \\
Mean \pm standard deviation & $42.01 \pm 20.58$ & $38.84 \pm 20.67$ \\
Correlation with RMD0 score (Spearman's) & $0.850(p<0.001)$ & $0.818(p<0.001)$ \\
Correlation with VAS for disability & $0.712(p<0.001)$ & $0.718(p<0.001)$ \\
Correlation with VAS for pain & $0.625(p<0.001)$ & $0.640(p<0.001)$ \\
\hline
\end{tabular}

ODI, Oswestry Disability Index; RMDQ, Rolland Morris disability questionnaire; VAS, Visual Analog Scale. 


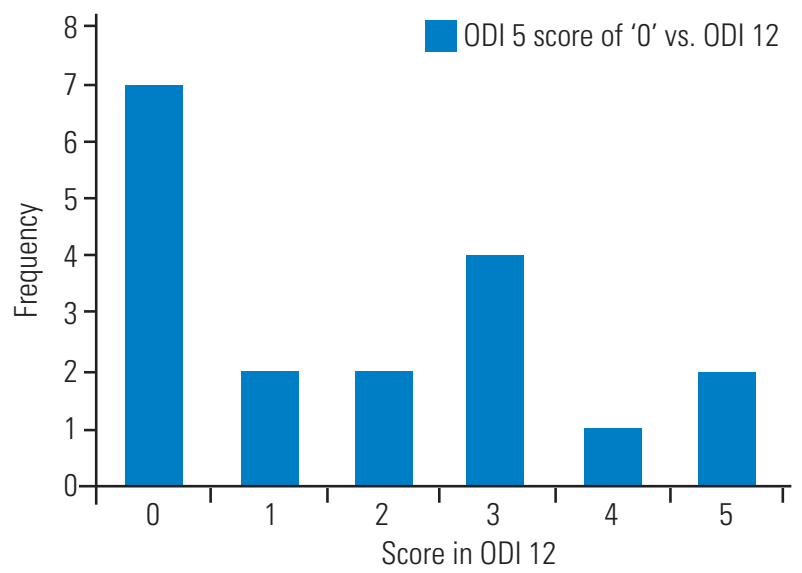

Fig. 1. Comparison of ODI 5 score of ' 0 ' with ODI 12 ( $N=18)$. ODI, Oswestry Disability Index.

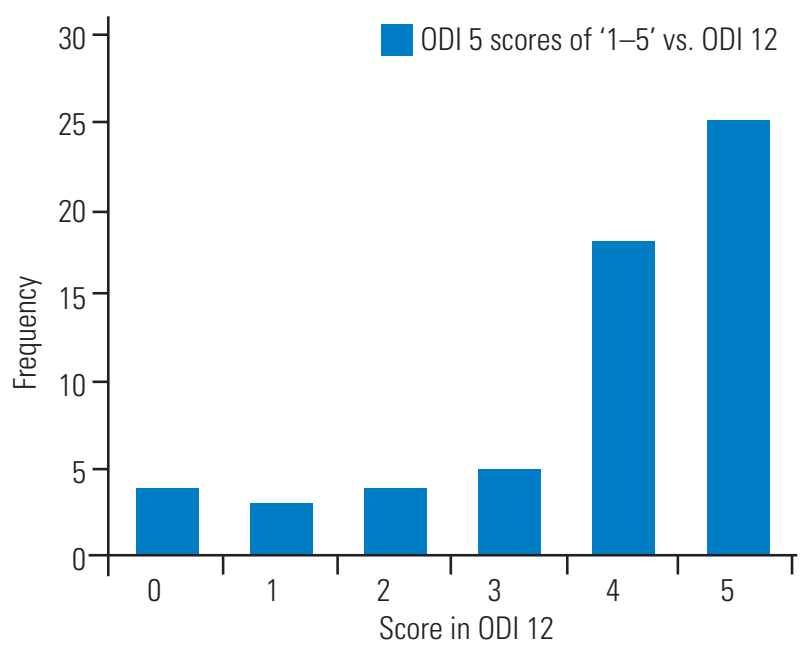

Fig. 2. Comparison of ODI 5 score of ' $1-5^{\prime}$ with $\mathrm{ODI} 12$ ( $\mathrm{N}=59$ ). ODI, Oswestry Disability Index.

floor) were later analyzed further. We aimed to determine whether patients who ticked the first response to ODI 5 could also sit comfortably on the floor. A comparison of an ODI 5 score of ' 0 ' with that for ODI 12 is shown in Fig. 1 , and a comparison of ODI 5 scores of ' $1-5$ ' with those of ODI 12 is shown in Fig. 2.

\section{Discussion}

The ODI is a very useful tool for assessing back-specific disabilities $[1,2]$, especially in patients with degenerative disc diseases. The ODI has been effectively used for the previous 35 years and is being used consistently during routine clinical visits as well as for predicting surgical treatment outcomes [3-7].
While the index was developed for the western population, in view of the cultural and social differences in India $[15,16]$, we believed that the scoring system could be adapted for India with some modifications. This study aimed to identify the modifications needed to adapt this questionnaire for patients in India and other developing countries. The senior author's earlier paper has highlighted the need for these modifications, including serious consideration toward the exclusion of weight lifting and sexual activity and addition of activities, such as sitting on the floor and squatting, which are relevant for the Indian population [14]. However, the authors did not make concrete plans to develop a modified questionnaire. Hence, this study was undertaken to modify the questionnaire to make it suitable to most developing countries because the prevalence of back pain-induced disability in these countries similar to each other.

Attempts have been previously made to modify the questionnaire in India. Sharma [21] proposed an additional question related to work status, while Nishant et al. [13] highlighted the change in pain. We felt these questionnaires are not developed based on actual methodology of questionnaire development [19]. Questionnaire development is a qualitative and quantitative process that needs to follow an approach as described by Gehlbach et al. [19]. To the best of our knowledge, this is the first study that attempted to modify an existing questionnaire using a standardized method of questionnaire development. Our free listing and qualitative discussion on each question with the patient helped us obtain qualitative data regarding the disability domains. Our qualitative analysis clearly showed that the questions related to sexual activity and weight lifting were irrelevant for most patients. While the statistical tests do not show any differences (internal consistency as well as correlation), the qualitative data are more important for understanding the significance of these questions. The questions were very well developed; therefore, it is possible that there would be correlation even if only a few patients answer these questions. Thus, we believed that the first step toward questionnaire development was to understand the disability domains of the target population. In our study, we found that majority of the patients felt that bending forward (67\%, $\mathrm{n}=20$ during free listing) was an important activity in their daily routine. Similarly, items regarding sexual activity and weight lifting were not answered by several (sexual activity $60 \%, \mathrm{n}=18$; weight lifting $16 \%, n=5$ ) patients. Many people reported that it is 
difficult to accurately score weight-lifting activity because their routine activities do not involve lifting weights [14]. Differentiating between moderate and heavy weights was also challenging. This information was obtained from the qualitative data from interviews. In the second part of the study that involved 88 patients, we found a discrepancy between number of people who reported that the question regarding weight lifting was irrelevant $(n=14)$ with respect to their daily routine and those who did not answer this question $(n=5)$. It is possible that many patients answered this question by speculation. The weight-lifting activity may be important for manual laborers (Table 3), but not in all the subjects. Further, the population comprised patients with degenerative disc disorders that affect the elderly; therefore, we found it appropriate to remove this question. Fischer and Johnson also found no correlation between the actual performance and scoring [7]. The research by our senior author also highlighted that people may speculate with regard to weight lifting [14]. Regarding the question on sexual activity, several authors have expressed similar reservations and developed modifications to the ODI, with replacement of the sexual activity clause with degrees of pain, employment/homemaking. Some researchers opted to remove it altogether [9-12]. Based on this literature review, we believed that the question related to sexual activity should be deleted. Although some studies have shown that sexual function is related to backache [9-12], given the cultural scenario, several patients from India may not answer this question, raising the chances for error. Thus, this question can be omitted. The disability related to sexual function may be directly related to other disabilities and may include a psychological wellbeing component.

In our attempt to trim the questionnaire to include only 10 questions, our next analysis aimed to identify any overlap in the questions. Responses to ODI 5 (sitting) and ODI 12 (sitting on the floor) were analyzed further. Our aim was to see whether those people who ticked the first response to question number 5 could also sit comfortably on the floor. A comparison of the ODI 5 score of ' 0 ' with that of ODI 12 is shown in Fig. 1 and the comparison of ODI 5 scores of ' $1-5$ ' with those of ODI 12 is shown in Fig. 2. This figure highlights that majority of the patients who scored ' 0 ' in ODI 5 also scored ' 0 ' in ODI 12 (38.8\%, $\mathrm{n}=7)$. All other scores of ODI 5 correlated with the 4 th and 5th response in ODI $12(72.8 \%, \mathrm{n}=43)$. Thus, most patients with a score of ' 0 ' in sitting could also sit on the floor, while those who could not sit on the floor received scores ranging from 1 to 5 , depending on their ability to sit in their favorite chair. Thus, it was possible to address the issue of 'sitting on the floor' in question number 5 itself.

After validating the questionnaire, we performed a reliability analysis with the standard ODI, RMDQ [18], and the VAS score. Almost all the questions showed good correlation, except for the question regarding sexual activity that showed a poor correlation (Table 5). All the new questions correlated well with the standard ODI (Tables $5,6)$. While the correlation statistics could show us how well the questions were framed, the validity and relevance of the questions should be decided using the qualitative data. Thus, we believe that the correlation should not be the sole criterion while selecting the 10 best questions. Although the correlation results can be used to prove that the questions are well framed, qualitative data, including the domains of disability should be considered while selecting the questions. We have already discussed the removal of the question regarding sexual activity and adding those related to sitting on the floor as one of the responses in question number 5. Among the remaining answered questions, the 13th question related to walking on uneven surface was not answered by many patients (Table 2), especially by those employed in clerical offices (Table 3). Moreover, a question on walking already exists; therefore, we may modify the first response to 'walking on any surface' while changing the second response to 'walking on uneven surface more than quarter of a mile.' The remaining responses will remain unchanged. Few patients felt that sitting on the floor was problematic because it involved getting up and sitting down, and this can be attributable to problems faced in bending forward. Thus, bending forward was an important question to be included; work status provided information about the overall disability status and thus was relevant to a patient's work status. The disability domains can vary slightly depending on the work status. Therefore, a question about work status is a flexible option which directly addresses the patient's work-related problems.

The questionnaire items can be divided into the following three categories: pain; common acts like sitting, bending, walking, and standing that are common denominators for most daily activities; and the third category that includes daily activities, such as traveling, sexual function, sport activities, work status, and personal care. While pain 
and routine daily activities may be the same for most patients, key functions, such as personal care, lifting weights, and traveling involve a combination of multiple tasks and may differ from person to person. Work status is an issue that incorporates most of the activities needed for that particular person and probably provide a flexible option to address the disabilities of different people with different work requirements. Weight lifting and traveling may vary from person to person; some individuals may not require these activities in their daily life. In our study, although we did not assess the amount of traveling done by each participant, we found that 18 out of 31 who performed manual labor considered traveling as irrelevant. Thus, we can consider weight lifting and social life/traveling as alternative questions for some patients. In sum, our proposed questionnaire involves seven questions, excluding sexual function, traveling, and weight lifting from the original ODI; further, we propose to add bending forward and work status to the modified ODI (Appendix 1). Questions regarding traveling and weight lifting can be changed based on the occupation; for a manual laborer, we can use weight lifting, while for others, we can use traveling. If neither of these activities is required, we still can calculate the ODI from the nine responses as described by Fairbank et al. [1] in their original description of the calculation of the final score when a patient has not answered all the questions.

The present study has several limitations. While our study has used a standard method of questionnaire development, including qualitative methods to understand the disability domains of a patient, the study was conducted in a single center that catered to patients from the locality. Hence, the questionnaire needs to be tested across multiple centers to improve its validity and efficacy $[22,23]$. Although we have analyzed the occupational profile of our patients that indicates even distribution of patients among different occupations, testing of the questionnaire can be considered with stratified sampling and different health care settings, such as primary health care, specialty service, and tertiary care. In addition, questions related to weight lifting and traveling need further testing on a larger sample in patients who are manual laborers and those whose work involves a lot of traveling. Future studies are also required to use this scoring system as a conditionspecific outcome assessment tool; most importantly, the questionnaire needs to be tested for its ability to show improvements in the treatment outcome. This is true for any questionnaire development; it would be ideal to have a report on development which we have already done and then subsequent studies on its use and psychomotor properties [24].

\section{Conclusions}

A modified disability questionnaire (modified from the ODI) prepared by adding two more questions related to bending forward and work status and deleting questions related to sexual activity and weight lifting or traveling (depending on patient occupation) can help assess the disability and function better in Indian patients with low back pain. However, these modifications need further testing at multiple centers, including the assessment of the changes in the scores following treatment.

\section{Conflict of Interest}

No potential conflict of interest relevant to this article was reported.

\section{Acknowledgments}

This study was partially funded by a university grant from Manipal Academy of Higher Education.

\section{ORCID}

Janardhana P. Aithala: https://orcid.org/0000-0002-6605-8331 Suraj Kumar: https://orcid.org/0000-0003-0583-3514

Shodhan Aithal: https://orcid.org/0000-0002-2534-1616

Shashidhar M. Kotian: https://orcid.org/0000-0001-5729-1337

\section{References}

1. Fairbank JC, Couper J, Davies JB, O'Brien JP. The Oswestry low back pain disability questionnaire. Physiotherapy 1980;66:271-3.

2. Bombardier C. Outcome assessments in the evaluation of treatment of spinal disorders: summary and general recommendations. Spine (Phila Pa 1976) 2000;25:3100-3.

3. Deyo RA, Battie M, Beurskens AJ, et al. Outcome measures for low back pain research: a proposal for standardized use. Spine (Phila Pa 1976) 1998;23:2003-13. 
4. Doleys DM, Klapow JC, Hammer M. Psychological evaluation in spinal cord stimulation therapy. Pain Rev 1997;4:189-207.

5. Turk DC, Marcus DA. Assessment of chronic pain patients. Semin Neurol 1994;14:206-12.

6. Fairbank JC, Pynsent PB. The Oswestry disability index. Spine (Phila Pa 1976) 2000;25:2940-52.

7. Fisher K, Johnston M. Validation of the Oswestry low back pain disability questionnaire, its sensitivity as a measure of change following treatment and its relationship with other aspects of the chronic pain experience. Physiother Theory Pract 1997;13:67-80.

8. Costa M, Marshman LA. Sex life and the Oswestry Disability Index. Spine J 2015;15:1225-32.

9. Hudson-Cook N, Tomes-Nicholson K, Breen A. A revised Oswestry disability questionnaire. In: Roland M, Jenner JR, editors. Back pain: new approaches to rehabilitation and education. Manchester: Manchester University Press; 1989. p.187-204.

10. Fritz JM, Irrgang JJ. A comparison of a modified Oswestry Low Back Pain Disability Questionnaire and the Quebec Back Pain Disability Scale. Phys Ther 2001;81:776-88.

11. Fairbank J. Use of Oswestry Disability Index (ODI). Spine (Phila Pa 1976) 1995;20:1535-7.

12. Daltroy LH, Cats-Baril WL, Katz JN, Fossel AH, Liang $\mathrm{MH}$. The North American Spine Society lumbar spine outcome assessment instrument: reliability and validity tests. Spine (Phila Pa 1976) 1996;21:741-9.

13. Nishant, Chhabra HS, Kapoor KS. New modified English and Hindi Oswestry disability index in low back pain patients treated conservatively in Indian population. Asian Spine J 2014;8632-8.

14. Aithala JP. Difficulties in using Oswestry Disability Index in Indian patients and validity and reliability of translator-assisted Oswestry Disability Index. J Orthop Surg Res 2015;10:90.

15. Mulholland SJ, Wyss UP. Activities of daily living in non-Western cultures: range of motion requirements for hip and knee joint implants. Int J Rehabil Res 2001;24:191-8.
16. Chopra A, Saluja M, Patil J, Tandale HS. Pain and disability, perceptions and beliefs of a rural Indian population: a WHO-ILAR COPCORD study. WHOInternational League of Associations for Rheumatology. Community Oriented Program for Control of Rheumatic Diseases. J Rheumatol 2002;29:614-21.

17. Kopec JA, Esdaile JM, Abrahamowicz M, et al. The Quebec Back Pain Disability Scale: measurement properties. Spine (Phila Pa 1976) 1995;20:341-52.

18. Roland M, Morris R. A study of the natural history of back pain: part I: development of a reliable and sensitive measure of disability in low-back pain. Spine (Phila Pa 1976) 1983;8:141-4.

19. Gehlbach H, Artino AR Jr, Durning S. AM last page: survey development guidance for medical education researchers. Acad Med 2010;85:925.

20. Boonstra AM, Schiphorst Preuper HR, Reneman MF, Posthumus JB, Stewart RE. Reliability and validity of the visual analogue scale for disability in patients with chronic musculoskeletal pain. Int J Rehabil Res 2008;31:165-9.

21. Sharma R. Epidemiology of musculoskeletal conditions in India. New Delhi: Indian Council of Medical Research ; 2012.

22. Vianin M. Psychometric properties and clinical usefulness of the Oswestry Disability Index. J Chiropr Med 2008;7:161-3.

23. McGirt MJ, Bydon M, Archer KR, et al. An analysis from the Quality Outcomes Database: part 1: disability, quality of life, and pain outcomes following lumbar spine surgery: predicting likely individual patient outcomes for shared decision-making. J Neurosurg Spine 2017;27:357-69.

24. Frost MH, Reeve BB, Liepa AM, Stauffer JW, Hays RD; Mayo/FDA Patient-Reported Outcomes Consensus Meeting Group. What is sufficient evidence for the reliability and validity of patient-reported outcome measures? Value Health 2007;10 Suppl 2:S94S105. 
Appendix 1. Modified disability questionnaire for Indian population

\section{Pain intensity}

$\square$ I have no pain at the moment

$\square$ Pain is very mild at the moment

$\square$ Pain is moderate at the moment

$\square$ Pain is fairly severe at the moment

$\square$ Pain is very severe at the moment

$\square$ Pain is the worst imaginable at the moment

\section{Personal care}

$\square$ I can look after myself normally without experiencing extra pain

$\square$ I can normally look after myself but it causes extra pain

$\square$ It is painful to look after myself, and I perform activities slowly and carefully

$\square$ I need some help but manage most of my personal care

$\square$ I need daily help for most aspects of self-care

$\square$ I cannot get independently dressed, and I wash myself with difficulty and stay in bed

\section{Walking}

$\square$ Pain does not prevent me from walking any distance on any surface $\square$ Pain prevents me from walking on uneven surfaces for more than

$1 / 4$ th of a mile, but I can walk $1 \mathrm{~km}$ or more on even-level surfaces

$\square$ Pain prevents me from walking for more than $1 \mathrm{~km}$

$\square$ Pain prevents me from walking for more than $500 \mathrm{~m}$

口 I can only walk using a stick or crutches

$\square \mathrm{l}$ am in bed most of the time

\section{Sitting}

口 I can sit in any chair or on the floor for as long as I like

口 I can only sit in my favorite chair for as long as I like

$\square$ Pain prevents me from sitting for more than 1 hour

$\square$ Pain prevents me from sitting for more than 30 minutes

$\square$ Pain prevents me from sitting for more than 10 minutes

$\square$ Pain prevents me from sitting at all

\section{Standing}

口 I can stand for as long as I want without extra pain

口 I can stand for as long as I want, but it causes extra pain

$\square$ Pain prevents me from standing for more than 1 hour

$\square$ Pain prevents me from standing for more than 30 minutes

$\square$ Pain prevents me from standing for more than 10 minutes

$\square$ Pain prevents me from standing at all

\section{Sleeping}

$\square$ My sleep is never disturbed by pain

$\square$ My sleep is occasionally disturbed by pain

口 I sleep for less than 6 hours because of pain

$\square$ I sleep for less than 4 hours because of pain

$\square$ I sleep for less than 2 hours because of pain

$\square$ Pain prevents me from sleeping at all

\section{Bending forward}

$\square$ Comfortable while picking objects from the floor by bending and can work for long periods

$\square$ Can bend and pick up objects from the floor but cannot do it consistently because of pain

$\square$ Can bend and pick up objects placed at knee level and work for long periods in that position

$\square$ Can bend and pick up objects placed at knee level occasionally

$\square$ Can bend and pick up objects placed at waist level only

$\square$ Cannot bend at all

\section{Work status}

$\square$ Able to do gainful work

$\square$ Able to do work, but with some pain, require medicines

$\square$ Able to do work, but with pain, medicines, and occasional work leave

$\square$ Irregular at work because of pain

$\square$ On loss of pay, but able to do household work and perform personal care

$\square$ Unable to do household work and am dependent

\section{Social life}

$\square$ My social life is normal and causes no extra pain

$\square$ My social life is normal, but I have more severe pain

$\square$ Pain has no significant effect on my social life apart from limiting my more energetic interests, such as sports

$\square$ Pain has restricted my social life, and I do not go out as often as I used to

$\square$ Pain has restricted my social life to my home

$\square$ I have no social life because of pain

\section{0-a) Traveling}

$\square$ I can travel anywhere without pain

$\square$ I can travel anywhere, but it causes extra pain

$\square$ Pain is severe, but I manage journeys over 2 hours

$\square$ Pain restricts me to journeys of less than 1 hour

$\square$ Pain restricts me to short, necessary journeys under 30 minutes

$\square$ Pain prevents me from traveling except to receive treatment

10-b) Lifting weights (to be used for patients who are manual laborers, in place of the earlier question on traveling)

$\square$ I can lift heavy weights without extra pain

$\square$ I can lift heavy weights but it causes extra pain

$\square$ Pain prevents me from lifting heavy weights off the floor, but I can manage if they are conveniently placed (e.g., on a table).

$\square$ Pain prevents me from lifting heavy weights, but I can manage light to medium weights if they are conveniently positioned

口 I can only lift very light weights

口 I cannot lift or carry anything at all 